\title{
Changing strategies of lung biopsies in diffuse lung diseases: the impact of video-assisted thoracoscopy
}

\author{
M. Ravini*, G. Ferraro**, B. Barbieri*, P. Colombo+, G. Rizzato*
}

\begin{abstract}
Changing strategies of lung biopsies in diffuse lung diseases: the impact of video-assisted thoracoscopy. M. Ravini, G. Ferraro, B. Barbieri, P. Colombo, G. Rizzato. CERS Journals Ltd 1998.

ABSTRACT: The aims of this report were: 1) to compare the strategy of bioptic approach in Italy during the last 4 yrs with a previous period; and 2) to compare efficacy and safety of video-assisted thorascopic lung biopsy (VTLB) versus OLB.

We retrospectively evaluated: 1) the strategy of the bioptic approach in the Milan Sarcoid Clinic in the years 1992-1995 (201 patients) versus 1988-1991 (197 patients); and 2) data from 65 VTLB procedures in the years 1992-1995 versus 68 OLB procedures in the years 1988-1991 performed in patients with diffuse lung disease.

It was found that the use of OLB (17-9\%), mediastinoscopy (15-5\%), and scalene node biopsy (20-7\%) decreased, whereas transbronchial biopsy (TBB) increased (11$17 \%$ ). VTLB biopsy is now performed in $17 \%$ of patients. VTLB compares favourably with OLB as there is less need for analgesia $(7.5 \pm 7.5$ versus $17.5 \pm 8.0$ methadone mg i.m.: p<0.001), lower blood loss $(61 \pm 58$ versus $156 \pm 84 \mathrm{~mL}$ in the first postoperative day: $p<0.001)$, and shorter postoperative stay $(4.7 \pm 1.6$ versus $5.7 \pm 1.4$ days: $p<0.001)$. Specimen adequacy $(98.6$ versus $98.5 \%)$ and diagnostic accuracy $(86.1 \%$ VTLB, versus 92.6\% OLB: $\mathrm{p}>0.05)$ were the same in the two groups.

In conclusion, video-assisted thoracoscopic lung biopsy is replacing both mediastinoscopy and open lung biopsy. It is at present the best option when a surgical procedure is required for histological confirmation of diffuse lung disease. Eur Respir J 1998; 11: 99-103.
\end{abstract}

*Thoracic Surgery, **Sarcoid Clinic and +Health Physics Dept, Niguarda Hospital, Milan, Italy.

Correspondence: G. Rizzato

Via Juvara 9

20129 Milan

Italy

Fax: 0039266102206

Keywords: Diffuse lung disease

interstitial lung disease

pulmonary biopsy

sarcoidosis

transbronchial biopsy

video-assisted thoracoscopy

Received: December 301996

Accepted after revision November 171997
Open lung biopsy (OLB) has for years been the "gold standard" for the invasive diagnosis of diffuse lung disease, with a diagnostic yield of $50-92 \%[1,2]$. However $1.8-70 \%$ mortality $[1,3]$, and $20-30 \%$ morbidity $[3,4]$ have been reported depending on chronicity and severity of the patients condition. Post-thoracotomy pain is the most frequent source of discomfort following OLB [5], and may also be a problem after minithoracotomy, because it is mainly caused by rib spreading.

Three comparative studies [5-7] have shown that videoassisted thoracoscopic lung biopsy (VTLB), despite the higher costs as a result of the use of disposable items, has a number of advantages over OLB, i.e. better visualization of the entire lung, reduced pain, reduced postoperative disability and shorter hospital stay. CARNOCHAN et al. [5] proposed that these advantages outweigh the increased cost in disposables. By contrast Molin et al. [8] have confirmed a very high diagnostic accuracy with VTLB, but no better patient benefit in terms of operating time, postoperative pain, chest-tube removal or postoperative stay.

These studies were all carried out in the USA or UK, while there are no other comparative reports. VTLB has been used in our hospital since January 1992. Since then, as suggested from the surgical procedures on patients referred to our clinic after a biopsy for interstitial lung disease, it has become apparent that in Italy mediastinoscopy, OLB and scalene biopsy are becoming less popular. More- over, diagnostic yield, hospital stay and other surgical parameters for VTLB in interstitial lung disease are unknown in Italy. The aims of this research were: 1) to compare the epidemiology of biopsies performed in the last 4 yrs, with a previous period, in patients referred to the Sarcoid Clinic of Milan; 2) to compare efficacy and safety of VTLB with OLB in our Hospital; and 3) to compare our results with the data reported in other literature.

\section{Patients and methods}

\section{Patients}

The Milan Sarcoidosis Clinic was opened in October 1978. Between October 1978 and December 1995 1,400 patients were examined. The final diagnoses are summarized in table 1 . Sarcoidosis was histologically proven in 782 cases. Table 2 shows the types of biopsy used.

VTLB was performed when the aetiological diagnosis of interstitial lung disease could not be obtained by bronchoalveolar lavage (BAL) and transbronchial biopsy (TBB) or by simpler extrapulmonary biopsies. Most patients had already undergone a nondiagnostic TBB elsewhere, before attending our clinic. Few patients refused bronchoscopy and preferred VTLB as a first choice biopsy. 
Table 1. - Milan Sarcoidosis Clinic 1978-1995: final diagnosis in 1,400 patients

\begin{tabular}{lr}
\hline Sarcoidosis, histologically proven & 782 \\
Sarcoidosis, not histologically proven & 221 \\
Other granulomatous disorders & 86 \\
Pulmonary fibrosis & 130 \\
Pneumoconiosis & 31 \\
Cancer & 31 \\
Uveitis & 60 \\
Erythema nodosum of unknown origin & 17 \\
Others & 40 \\
\hline
\end{tabular}

Table 2. - Type of biopsies performed in 782 patients with sarcoidosis, October 1978-December 1995

\begin{tabular}{lcl}
\hline & $\mathrm{n}$ & \multicolumn{1}{c}{ Biopsy type $\mathrm{n}$} \\
\hline Lung & 238 & $\begin{array}{l}\text { Open 86, TBB 118, } \\
\text { VTLB 34 } \\
\text { Lymph nodes }\end{array}$ \\
& 296 & $\begin{array}{l}\text { Mediastinoscopy 104 } \\
\text { Scalene 89 } \\
\text { Peripheral 102* }\end{array}$ \\
& & Retroperitoneal 1 \\
Liver & 31 & 13 on scar \\
Skin & 82 & \\
Kveim & 25 & \\
Multiple & 78 & \\
Others** & 32 & \\
\hline
\end{tabular}

TTB: transbronchial biopsy; VTLB: video-assisted thoracoscopic biopsy. *: neck or supraclavear six, groin 13, axilla six; **: parotis gland, spleen, kydney, muscle, bone, joints, central nervous system, etc.

We re-examined the records of the patients undergoing VTLB for a diffuse lung disease during the period 19921995. The series included 70 subjects, 34 from the Milan Sarcoidosis Clinic and 36 referred from other divisions of the hospital or from other hospitals. This group was compared to a historical group of 68 patients who underwent OLB for diffuse interstitial lung disease during the period 1988-1991.

\section{Methods}

$V T L B$. Patients were intubated by using a double-lumen endotracheal tube and were placed in lateral decubitus position. A $12 \mathrm{~mm}$ chest wall incision was made in the 6th intercostal space on the midaxillary line and a $10.5 \mathrm{~mm}$ trocar inserted into the pleural cavity while the ipsilateral lung was deflated. A $0^{\circ} 10 \mathrm{~mm}$ rigid thoracoscope with camera attached to a video monitor was then inserted through the port and lung and pleura were inspected. Two additional trocars $(5.5 \mathrm{~mm}$ and $11.5 \mathrm{~mm})$ were then introduced under direct thoracoscopic vision, usually into the 5th intercostal space on the anterior and posterior axillary line respectively. Following exploration, with the lung partially inflated, a suitable biopsy site was chosen. An endoscopic stapling device (End-Gia-Autosuture, US Surg. Corp. Norwalk, CT, USA) was inserted through the posterior port $(11.5 \mathrm{~mm})$ and fired, transecting the lung with placement of a double staple line. In our series many patients had a clinical history and a radiological pattern suggesting stage I or II sarcoidosis. Usually when mediastinal nodes were enlarged a biopsy of one or more of these was performed, in addition to the lung biopsy. After taking the specimen(s), two of the ports were closed and the third was used for a pleural underwater drain. We chose to biopsy the lung that appeared more impaired. When pulmonary lesions appeared equally distributed on chest radiography or computed tomography (CT) scan (a very frequent condition), surgery was performed on the right lung for anatomical reasons. Thus 57 procedures were performed on the right lung, and eight on the left. Blood loss refers to the amount of blood collected from pleural drainage in the first $24 \mathrm{~h}$ following the surgical procedure. Analgesia in the postoperative period was obtained by administering methadone $100 \mathrm{mg}$ i.m., according to patients requirements.

$O L B$. The patient, under general anaesthesia and intubated with a double lumen endotracheal tube, was positioned in the lateral decubitus with ipsilateral arm abduced at $90^{\circ}$. A $6 \mathrm{~cm}$ axillary thoracotomy was performed from the $3 \mathrm{rd}$ to the 4th rib, along the anterior margin of the latissimus dorsi muscle. On entering the pleural cavity, the lung was inspected and the site of biopsy chosen. Biopsy was performed using an automatic stapling device. A nodal biopsy was also obtained when enlarged mediastinal lymphnodes were present. After inspection of the transected lung for bleeding and air leak, a chest-tube was inserted through a separate incision and was connected to an underwater drainage system. The thoracotomy was then closed. Sixty one patients underwent surgery on the right lung, and seven on the left.

\section{Statistical analysis}

Results are expressed as mean \pm SD. Statistical analysis of the data was performed using two-tailed unpaired Student's t-test, or Chi-squared Fisher's exact test when appropriate. A type I error of 5\% and type II error of $10 \%$ was assumed.

\section{Results}

\section{Comparison of site of biopsy between two periods}

In the last 4 yrs (1992-1995), a final histological diagnosis of sarcoidosis was reached in 201 patients referred to the Milan Sarcoidosis Clinic. The biopsies performed in these patients are described in table 3 .

Table 3. - Biopsies in the period 1992-1995: 201 patients with sarcoidosis

\begin{tabular}{lrrrcccc}
\hline & VTLB & TBB & OLB & SNB & MDB & Others* & Total \\
\hline Stage I & 19 & 7 & 3 & 6 & 7 & 27 & 69 \\
Stage II & 8 & 8 & 10 & 4 & 2 & 21 & 53 \\
Stage III & 7 & 15 & 4 & 5 & 2 & 18 & 51 \\
Stage 0 & - & 1 & 1 & - & - & 26 & 28 \\
Total & 34 & 31 & 18 & 15 & 11 & 92 & 201 \\
\hline
\end{tabular}

VTLB: video-assisted thoracoscopic lung biopsy; TBB: transbronchial biopsy; OLB: open lung; SNB: scalene node biopsy; MD: mediastinoscopy; *: including multiple biopsies. 


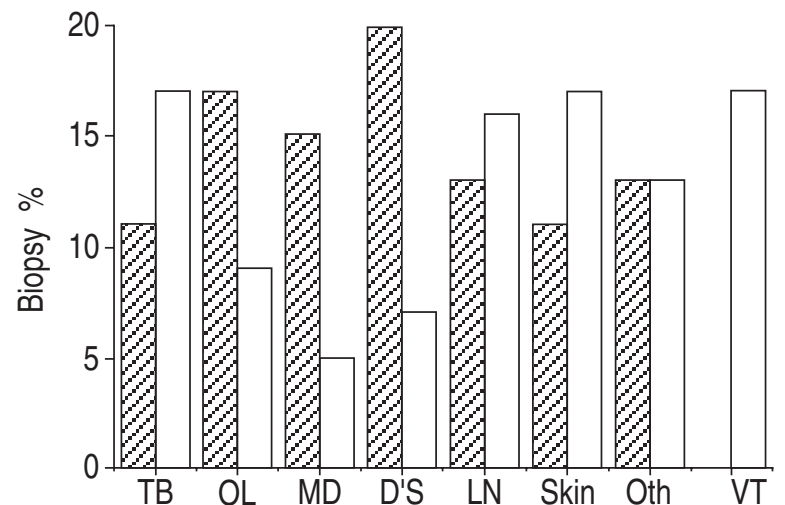

Fig. 1. - Percentage of different biopsies performed in patients with a final diagnosis of sarcoidosis. 62 : 1988-1991, 197 patients; $\square$ : 19921995, 201 patients. TB: transbronchial; OL: open lung; MD: mediastinoscopy; D'S: scalene node; LN: other extrathoracic lymphnodes; VT: video-assisted thoracoscopy; Oth: others (multiple in most cases).

Figure 1 compares the data of the period 1992-1995 (201 patients) versus the period 1988-1991 (197 patients). The use of OLB (17-9\%), mediastinoscopy (15-5\%), and scalene node biopsy (20-7\%) declined, whereas TBB increased $(11-17 \%)$. VTLB biopsy is now performed in $17 \%$ of patients.

\section{Comparison of $V T L B$ versus $O L B$}

In the last 4 yrs, 70 patients with interstitial lung disease underwent VTLB with or without mediastinal node biopsy. Five of these 70 were converted to thoracotomy during surgery because of extensive pleural adhesions, so that 65 patients were considered for the present study. The characteristics of the two populations who underwent VTLB during the last $4 \mathrm{yrs}$, and OLB in the previous period are shown in table 4 . In patients with a final diagnosis of sarcoidosis the distribution according to their chest radiography at the time of surgery was also similar in the two groups (see below).

Surgical results are described in table 5. Surgery time was shorter in OLB patients, whereas estimated blood loss, analgesia and postoperative stay were better in VTLB patients. There were no deaths.

Table 4. - Characteristics at the time of surgery

\begin{tabular}{lccc}
\hline & $\begin{array}{c}\text { VTLB } \\
(\mathrm{n}=65)\end{array}$ & $\begin{array}{c}\text { OLB } \\
(\mathrm{n}=68)\end{array}$ & $\begin{array}{c}\text { Signifi- } \\
\text { cance* }\end{array}$ \\
\hline Male/Female & $33 / 32$ & $29 / 39$ & NS \\
Mean age yrs & $39 \pm 13$ & $40 \pm 13$ & NS \\
VC \% pred & $92 \pm 16$ & $90 \pm 12$ & Ns \\
$\mathrm{FEV} 1 \%$ pred & $89 \pm 16$ & $90 \pm 9$ & NS \\
$T \mathrm{~L}, \mathrm{CO} \%$ pred & $76 \pm 17$ & $72 \pm 12$ & NS \\
$P_{\mathrm{a}, \mathrm{O}_{2}} \mathrm{kPa}$ & $12.5 \pm 2.1$ & $12.6 \pm 2.7$ & NS \\
$P_{\mathrm{a}, \mathrm{CO}_{2} \mathrm{kPa}}$ & $5.2 \pm 0.7$ & $5.1 \pm 0.4$ & NS \\
Long-term $\mathrm{O}_{2}$ therapy & 0 & 0 & NS \\
Corticosteroid therapy & 3 & 5 & Ns \\
\hline
\end{tabular}

VTLB: video-assisted thoracoscopic lung biopsy; OLB: open lung biopsy; VC: vital capacity; *: type 1 error of $5 \%$ and a type II error of $10 \%$ was assumed. $1 \mathrm{mmHg}=0.133 \mathrm{kPa} ; \%$ pred: $\%$ of predicted value; FEV1: forced expiratory volume in one second; $T \mathrm{~L}, \mathrm{CO}$ : transfer factor of the lung for carbon monoxide; $P \mathrm{a}, \mathrm{O}_{2}$ : arterial oxygen tension; $\mathrm{Pa}_{\mathrm{a}} \mathrm{CO}_{2}$ : arterial carbon dioxide tension. Ns: nonsignificant.
Table 5. - Surgical results

\begin{tabular}{lccc}
\hline & VTLB & OLB & p-value \\
\hline $\begin{array}{l}\text { Surgery time min } \\
\text { Analgesia (i.m. methadone) } \\
\text { mg.patient }\end{array}$ & $64 \pm 24$ & $52 \pm 41$ & $<0.01$ \\
$\begin{array}{l}\text { Blood loss mL } \\
\text { (first postoperative day) }\end{array}$ & $7.5 \pm 7.5$ & $17.5 \pm 8.0$ & $<0.001$ \\
Pleural drainage days & $2.4 \pm 1.2$ & $2.3 \pm 1.0$ & NS \\
Postoperative stay days & $4.7 \pm 1.6$ & $5.7 \pm 1.4$ & $<0.001$ \\
$\begin{array}{l}\text { Biopsy number lung/nodes } \\
\text { Specimen adequacy \% }\end{array}$ & $63 / 98$ & $68 / 101$ & Ns \\
Diagnostic accuracy \% & 98.5 & 98.6 & Ns \\
\hline
\end{tabular}

VTLB: video-assisted thoracoscopic lung biopsy; OLB: open lung biopsy.

Complications occurred as follows:

1) Patients undergoing VTLB. One prolonged lymph effusion following mediastinal node biopsy, needing pleural drainage of 9 days: three cases of air leak (one of them with subcutaneous emphysema): two cases of partial lung collapse after removing drainage. A $57 \mathrm{yr}$ old female with idiopathic pulmonary fibrosis developed severe pneumonia with respiratory failure on the sixth postoperative day. Transfer to the intensive care unit (ICU) was needed for 4 days, before returning to the general ward.

2) Patients undergoing OLB. Two patients had incomplete lung re-expansion with persistent air leak, requiring prolonged pleural drainage for 6 and 17 days respectively. Six patients developed wound dehiscence.

A final diagnosis was reached as follows:

1) VTLB group (65 patients). In two patients the specimen was "nondiagnostic", and in seven others "nonspecific interstitial fibrosis". Thus a final diagnosis was reached in 56 patients: 47 sarcoidosis, five idiopathic pulmonary fib-rosis, two bronchiolitis obliterans with organizing pneumonia (BOOP), one interstitial lymphocytic pneumonia and one histiocytosis X. Sarcoidosis was stage I in 22 patients, stage II in 16 patients, and stage III in nine pat-ients. In four patients with stage I sarcoidosis, the lung specimen was nondiagnostic, and the diagnosis was obtained on mediastinal lymphnode sample.

2) OLB group (68 patients). In two patients the specimen was "nondiagnostic" and in three others "nonspecific interstitial fibrosis". Thus a final diagnosis was reached in 63 patients; 55 had sarcoidosis; four idiopathic pulmonary fibrosis; and one each for histiocytosis X, eosinophilic pneumonia, silicosis and tuberculosis. Sarcoidosis was stage I in 20 patients, stage II in 20 patients and stage III in 15 patients. In four patients with stage I sarcoidosis, the lung specimen was nondiagnostic, and diagnosis was obtained with biopsy of the mediastinal lymphnodes.

\section{Discussion}

This study showed that the use of OLB, mediastinoscopic biopsy and scalene node biopsy was declined, and that the use of TBB has increased in recent years as bioptic approaches in diffuse lung disease. The results further show that VTLB compares favourably with OLB as there is less need for analgesia, a lower blood loss, and a shorter postoperative stay. In addition, cosmetics results are better. Minithoracotomy provides only limited visualization of the lung and the biopsy specimen is usually taken from 
the most accessible area. On the contrary, VTLB permits a complete visual inspection of the lung, allowing the optimal segment for biopsy. In spite of this advantage, specimen adequacy and diagnostic accuracy were the same in the two groups. The simplest explanation may be that a diffuse process with granuloma formation, such as sarcoid, would probably be free from sampling error.

Our surgical results are similar to those described by other comparative reports [5-7], even if the different characteristics of patients may explain some differences. In the study of BENSARD et al. [7]: the forced vital capacity (FVC) of VTLB patients was $62 \pm 3$ versus $90 \pm 12 \%$ of predicted value in the present study; $55 \%$ of their VTLB patients were on steroids, as opposed to only five of 68 patients in the present series; $14 \%$ required supplemental oxygen, whereas no such situation arose in the present study. Moreover in the present study there were 22 patients (versus 0) with Stage I sarcoidosis, i.e. clear lungs radiograph. Thus, the patients in the study by BeNSARD et al. [7] were more severely impaired. This explains why $36 \%$ required transfer to the ICU, whereas only one pat-ient in the present study required such transfer. For the same reason, in the study by FeRSON et al. [6] there were three deaths, compared with none in the present study.

In comparison to the results given by FERSON et al. [6], results in the present study gave a better diagnostic yield. In the study by FERSON et al. [6] 21 of 47 patients had nonspecific interstitial fibrosis, whereas only five patients had this diagnosis in the present study. This is probably due to higher numbers of patients with sarcoidosis in the present series. In a large series of 467 patients with diffuse lung diseases, the overall diagnostic accuracy of VTLB was $86 \%$, but differed depending upon the underlying disease; the best results were obtained in sarcoidosis, with an accuracy of $98 \%$ [9].

The time necessary for pleural drainage was the same in the two groups in the present study and depended on the complete reinflation of the lung after surgery. In Bensard's patients [7] the time necessary for pleural drainage was shorter (1.3 \pm 0.2 versus $3.2 \pm 0.3$ days in the OLB group). FERSON et al. [6] and Molin et al. [8] have observed the same trend. The difference with respect to other authors may be due to our policy to maintain pleural drainage for at least $24 \mathrm{~h}$ after chest radiography demonstrated complete lung reinflation.

Similar to two other studies [6,7], the mean operative time was longer in the VTLB $(64 \pm 24 \mathrm{~min})$ versus OLB group $(52 \pm 21)(\mathrm{p}<0.01)$ in the present study, but the time decreased as additional experience was gained, and has been $54 \pm 37$ min for the last 20 patients who underwent VTLB. As such when time is considered for the last 20 performances, the operative time is comparable in the two groups ( $54 \pm 37$ versus $52 \pm 21 \mathrm{~min}$ : $\mathrm{p}>0.05)$.

One limitation is that a cost effective analysis was not among the aims of the present study. Molin et al. [8] have shown that VTLB results in higher procedure-related costs, with no advantage for the patient. By contrast other comparative studies [5-7] have shown that reduced postoperative disability and consequently the decreased hospital stay outweigh the increased cost of disposables. Thus, VTLB is considered to be cost-effective in the majority of the published studies.

Another limitation of the present study is that the population consisted mainly of patients suffering from sarcoi- dosis and the data do not permit a reliable evaluation of the VTLB approach in other diffuse lung diseases. However VTLB appeared to be a good enough approach in many other interstitial lung diseases in others studies [5-8].

A third limitation, both in the present study and others [5-8], is that all are retrospective and not randomized. A recent clinical commentary [10] concluded that VTLB will perhaps be accepted as the procedure of choice for lung biopsy in interstitial lung disease in the near future, even if its effectiveness needs to be confirmed by prospective studies. We agree with the conclusion of RAGHU [10], that, at present, VTLB should become a routine procedure in all institutions.

As regards the strategy of biopsy, the data do not reflect our policy of bioptic approach as they include all the patients who were referred to the Milan Sarcoidosis Clinic, including 300 of 398 patients $(75.3 \%)$, during the years 1988-1995, in whom a histological diagnosis had already been made elsewhere, prior to referral. Can these data be considered representative of the policy in the whole country? Probably not: the Milan Sarcoidosis Clinic is still the only centre recognized by the Italian Government, thereby representing a referral centre for the most difficult cases, which come from all over the country. In the Thoracic Surgery Dept, scalene node biopsy has not been performed in the last 8 yrs. However, $20 \%$ of the patients between the years 1988-1991, and 7\% during the years 1992-1995 underwent scalene node biopsy elsewhere, which represents a declining trend in the use of this technique throughout the country. The increase in peripheral lymph node biopsy and skin biopsy probably reflects the tendency to use less invasive procedures. Ninety eight patients were referred to the Milan Sarcoidosis Clinic with no previous histology: most had been subjected to a negative transbronchial biopsy elsewhere, suggesting the need for a more invasive approach. This may explain why in the last 4 yrs the same number (17\% of the total) of TBBs and VTLBs have been performed. Elsewhere, TBB diagnostic yields of 57-90\% have been reported in sarcoidosis [1114], which means that one should expect the number of TBBs to be at least double that of VTLB.

In the past transcervical mediastinoscopy was commonly performed in suspected cases of sarcoidosis with a $90 \%$ diagnostic accuracy, but with major complications (haemorrhage requiring sternotomy) in two of the most recent 25 cases. Following the introduction of VTLB, this technique has been adopted to perform mediastinal node and lung biopsy, by the same procedure but with less risk to the patient. Mediastinoscopy has been reserved for the diagnosis of lymphoproliferative disease and lung cancer staging. Thus, the 5\% mediastinoscopy of the last 4 yrs is totally due to procedures carried out in other centres. RAGHU [10] suggests that the finding of mediastinal ad-enopathy will prompt mediastinoscopy prior to VTLB. However, to our knowledge no studies have been carried out comparing mediastinoscopy and VTLB in the diag-nosis of sarcoidosis. Recently, in a study on 114 patients with mediastinal solid masses and/or lymphnodes (12 with sarcoidosis), Gossor et al. [15] have shown that the diagnostic yield of mediastinoscopy is comparable to thoracoscopy, while complication rates and length of hospital stay of patients undergoing mediastinoscopy are significantly lower. They concluded that VTLB should be indicated only for lesions that are not within the reach of the medi- 
astinoscope or when multiple biopsy specimens are necessary. The question however remains open, because VTLB permits biopsy of both lung and lymph nodes.

In conclusion, video-assisted thoracoscopic lung biopsy is replacing open lung biopsy. It is at present the best option when a surgical procedure is required for histological confirmation of diffuse lung disease.

\section{References}

1. Chuang M, Raskin J, Krellenstein D, Teirstein A. Bronchoscopy in diffuse lung disease: evaluation by open lung biopsy in nondiagnostic transbronchial lung biopsy. Ann Otol Rhinol Laryngol 1987; 96: 654-657.

2. Wall C, Gaensler E, Carrington C, Hayes J. Comparison of transbronchial and open biopsies in chronic infiltrative lung disease. Am Rev Respir Dis 1981; 123: 280-295.

3. Warner D, Warner M, Divertie M. Open lung biopsy in patients with diffuse pulmonary infiltrates and acute respiratory failure. Am Rev Respir Dis 1988; 137: 90-94.

4. Hall T, Hutchins G, Baker R. A critical review of the use of open lung biopsy in the management of the oncologic patient with acute pulmonary infiltrates. Am J Clin Oncol 1987; 10: 249-252.

5. Carnochan F, Walker W, Cameron E. Efficacy of video assisted thoracoscopic lung biopsy: an historical comparison with open lung biopsy. Thorax 1994; 49: 361-363.

6. Ferson P, Landreneau R, Dowling R, et al. Comparison of open versus thoracoscopic lung biopsy for diffuse infil- trative pulmonary disease. J Thoracic Cardivasc Surg 1993; 106: 194-199.

7. Bensard D, McIntyre R, Waring B, Simon J. Comparison of video thoracoscopic lung biopsy to open lung biopsy in the diagnosis of interstitial lung disease. Chest 1993; 103: 765-770.

8. Molin LJ, Steinberg JB, Lanza LA. VATS increases costs in patients undergoing lung biopsy for interstitial lung disease. Ann Thorac Surg 1994; 58: 1595-1598.

9. Loddenkemper R, Boutin C. Thoracoscopy: present diagnostic and therapeutic indications. Eur Respir J 1993; 6: 1544-1555.

10. Raghu R. Interstitial lung disease: a diagnostic approach. Am J Resp Crit Care Med 1995; 151: 909-914.

11. Koerner S, Sakowitz A, Appelman R, et al. Transbronchial lung biopsy for the diagnosis of sarcoidosis. New Engl J Med 1975; 293: 268-270.

12. Poe R, Israel R, Utell M, Hall W. Probability of a positive transbronchial lung biopsy result in sarcoidosis. Arch Intern Med 1979; 139: 761-763.

13. Koontz C, Joyner L, Nelson R. Transbronchial lung biopsy via the fiberoptic bronchoscope in sarcoidosis. Ann Intern Med 1976; 85: 64-66.

14. Mitchell DM, Mitchell DN, Collins J, Emerson C. Transbronchial lung biopsy through fibreoptic bronchoscope in diagnosis of sarcoidosis. Br Med J 1980; 280: 679-681.

15. Gossot D, Toledo L, Fritsch S, Celerier M. Mediastinoscopy vs Thoracoscopy for mediastinal biopsy. Results of a prospective nonrandomized study. Chest 1996; 110 : $1328-1331$. 